\title{
On the Geometry of Differential Equations Associated with the Theta-zerovalue
}

By

\author{
Jun-ichi YosHIDA *
}

\section{\$1. Introduction}

In [4], Kashiwara-Kawai-Takei made a deep analysis of the structure of the cohomology groups associated with the Riemann theta-zerovalue:

$$
\vartheta(t)=\sum_{\nu \in \mathbf{Z}^{n}} \exp \pi \sqrt{-1}\langle\nu, t \nu\rangle
$$

with $t$ being a symmetric complex $n \times n$ matrix and the imaginary part of $t$ being positive definite, when $n=2$. Their approach is to analyse the structure of linear differntial equations of infinite order which the theta-zerovalue satisfies. This approach was first advocated by Sato ([7]) and later pursued by Sato, Kashiwara, Kawai and Takei ([4],[6], [8], [9], etc.).

In this paper we present some geometric results which are needed to extend the results of [4] for an arbitrary $n$. Although we have not yet obtained the complete generalization in this paper, we plan to discuss the more analytic aspect of the problem, using the algebraic and geometric results shown here.

The plan of this paper is as follows; in $\S 2$, we first introduce $(n+1) \times(n+$ 1) matrices $P_{1}, \ldots, P_{n}, Q_{1}, \ldots, Q_{n}$ of linear differntial operators of finite order such that the following relations hold:

$$
\left(\exp P_{l}-I\right)\left(\begin{array}{c}
\vartheta(t) \\
0 \\
\vdots \\
0
\end{array}\right)=0
$$

Communicated by T. Kawai, January 9, 1995. Revised February 6, 1995.

1991 Mathematics Subject Classification (s): 11F27. 35A27.

* Research Institute for Mathematical Sciences, Kyoto University, Kyoto 606-01, Japan 


$$
\left(\exp Q_{l}-I\right)\left(\begin{array}{c}
\vartheta(t) \\
0 \\
\vdots \\
0
\end{array}\right)=0
$$

for $l=1, \ldots, n$. Here $I$ denotes the identity matrix of size $(n+1)$ and exp $P_{l}$ (resp., $\exp Q_{l}$ ) denotes the operator $\sum_{j=0}^{\infty} P_{l}^{j} / j$ ! (resp., $\left.\sum_{j=0}^{\infty} Q_{l}^{j} / j !\right)$, which turns out to be a matrix of linear differential operators of infinite order. Then $\exp P_{l}$ and $\exp Q_{l}$ act on the sheaf of holomorphic functions as sheaf homomorphisms. An interesting problem is to characterize the theta-zerovalue by the local conditions such as (1) and (2). Unfortunately, however, the simultaneous equations (1) and (2) do not make sense as they stand, because exp $P_{l}$ 's and $\exp Q_{m}$ 's do not commute. Hence the first step is to find out the correct auxiliary linear differential equations of finite order with which (1) and (2) make sense. Following the idea of Kawai [6], we construct the auxiliary equations by making use of the iterated commutators $\left[Q_{l}, Q_{m}\right]$ and $\left[Q_{k},\left[Q_{l}, Q_{m}\right]\right]$ which are denoted by $R_{[l, m]}$ and $S_{[k, l, m]}$, respectively. Actually we can verify that the addition of the differntial equations

$$
R_{[l, m]}\left(\begin{array}{c}
\vartheta(\mathrm{t}) \\
0 \\
\vdots \\
0
\end{array}\right)=0
$$

$$
S_{[k, l, m]}\left(\begin{array}{c}
\vartheta(\mathrm{t}) \\
0 \\
\vdots \\
0
\end{array}\right)=0
$$

suffices for our purpose (Theorem 2.2 and Definition 2.3). We can then discuss the characteristic set of the simultaneous equations (1), (2), (3) and (4). The description of the characteristic set is done by using a result of Aoki [1] (cf. [2] also) and it is a natural generalization of the corresponding result in the case $n$ $=2([4])$. The result indicates that the domain $X_{+}=\{t$ is a symmetric complex $n \times n$ matrix and the imaginary part of $t$ is positive definite $\}$ plays a special role in the study of the system of equations (1), (2), (3) and (4). In fact, once we get the well-defined system of equations (1), (2), (3) and (4), a result of [8] tells us that any holomorphic solution of the system on $X_{+}$is equal to $(\vartheta(t), 0$, $\ldots, 0)^{\prime}$ up to a constant factor. Here and in what follows we denote the transposed matrix of a matrix $A$ by $A^{\prime}$. Having this fact in mind, we study in $\S 3$ how 
the operators $P_{l}, Q_{m}, R_{[l, m]}$ and $S_{[k, l, m]}$ are changed under the imaginary transformation, i.e., $t \mapsto-t^{-1}$, and we show that (1), (2), (3) and (4) remain essentially unchanged under the imaginary transformation. Thus we deduce the coincidence of $\vartheta(t)$ and its imaginary transform by their local properties, that is, by the fact they satisfy a system of linear differential equations.

The author would like to express his heartiest thanks to Professor Kawai, Professor Aoki and Professor Takei for their many valuable suggestions.

\section{§2. Construction of a System of Linear Differential Equations and its Characteristic Set}

Let $X$ be the space of symmetric $n \times n$ complex matrices $\left(t_{i j}\right)_{1 \leq i, j \leq n}$ and let $\partial_{i j}$ denote the vector field $\frac{\partial}{\partial t_{i j}}+\frac{\partial}{\partial t_{j i}}$ on $X$ so that

$$
\partial_{i j} t_{k l}=\delta_{i k} \delta_{j l}+\delta_{i l} \delta_{j k} \quad \text { for } \quad 1 \leq i, j, k, l \leq n+1
$$

holds where $\delta_{i j}$ is Kronecker's delta. Using these notations, we introduce the following $(n+1) \times(n+1)$-matrices of differential operators $P_{l}$ and $Q_{l}$ for $l=1, \ldots, n$ after Kawai [6]:

$$
\begin{aligned}
& P_{l}=\left(\begin{array}{cccccc}
0 & t_{l 1} & \cdots & t_{l j} & \cdots & t_{l n} \\
2 \pi \sqrt{-1}\left(\delta_{l 1}+\sum_{k=1}^{n} t_{l k} \partial_{k 1}\right) & 0 & \cdots & 0 & \cdots & 0 \\
\vdots & \vdots & & \vdots & & \vdots \\
2 \pi \sqrt{-1}\left(\delta_{l j}+\sum_{k=1}^{n} t_{l k} \partial_{k j}\right) & 0 & \cdots & 0 & \cdots & 0 \\
\vdots & \vdots & & \vdots & & \vdots \\
2 \pi \sqrt{-1}\left(\delta_{l n}+\sum_{k=1}^{n} t_{l k} \partial_{k n}\right) & 0 & \cdots & 0 & \cdots & 0
\end{array}\right), \\
& Q_{l}=\left(\begin{array}{cccccc}
0 & \delta_{l 1} & \cdots & \delta_{l j} & \cdots & \delta_{l n} \\
2 \pi \sqrt{-1} \partial_{l 1} & 0 & \cdots & 0 & \cdots & 0 \\
\vdots & \vdots & & \vdots & & \vdots \\
2 \pi \sqrt{-1} \partial_{l j} & 0 & \cdots & 0 & \cdots & 0 \\
\vdots & \vdots & & \vdots & & \vdots \\
2 \pi \sqrt{-1} \partial_{l n} & 0 & \cdots & 0 & \cdots & 0
\end{array}\right) .
\end{aligned}
$$

These operators are related to the Riemann theta-zerovalue defined by

$$
\vartheta(t)=\sum_{\nu \in \mathbb{Z}^{n}} \exp \pi \sqrt{-1}\langle\nu, t \nu\rangle
$$


with $t$ being in the set

$$
X_{+}=\{t \in X \mid \text { the imaginary part of } t \text { is positive definite }\}
$$

in the following manner.

Lemma 2.1. The $(n+1)$-column vector $(\vartheta(t), 0, \ldots, 0)^{\prime}$ satisfies the following equations:

$$
\left(\exp P_{l}-I\right)\left(\begin{array}{c}
\vartheta(t) \\
0 \\
\vdots \\
0
\end{array}\right)=0
$$

$$
\left(\exp Q_{l}-I\right)\left(\begin{array}{c}
\vartheta(t) \\
0 \\
\vdots \\
0
\end{array}\right)=0
$$

for $1 \leq l \leq n$ on $X_{+}$.

Proof. For $\nu=\left(\nu_{1}, \ldots, \nu_{n}\right)^{\prime}$ in $\mathbb{R}^{n}$ let $e_{\nu}(t)$ denote the following $(n+1)-$ column vector:

$$
\left(\begin{array}{c}
\exp \pi \sqrt{-1}\langle\nu, t \nu\rangle \\
2 \pi \sqrt{-1} \nu_{1} \exp \pi \sqrt{-1}\langle\nu, t \nu\rangle \\
\vdots \\
2 \pi \sqrt{-1} \nu_{n} \exp \pi \sqrt{-1}\langle\nu, t \nu\rangle
\end{array}\right)
$$

It is then clear that

$$
\sum_{\nu \in \mathbf{Z}^{n}} e_{\nu}(t)=\left(\begin{array}{c}
\vartheta(t) \\
0 \\
\vdots \\
0
\end{array}\right)
$$

holds for $t$ in $X_{+}$.

Let us first prove (8). A straightforward computation shows 


$$
Q_{l} e_{\nu}(t)=2 \pi \sqrt{-1} \nu_{l} e_{\nu}(t)
$$

for each $\nu$ in $\mathbb{R}^{n}$ and $1 \leq l \leq n$. Hence we have

$$
\begin{aligned}
\exp Q_{l} e_{\nu}(t) & =\exp \left(2 \pi \sqrt{-1} \nu_{l}\right) e_{\nu}(t) \\
& =e_{\nu}(t)
\end{aligned}
$$

if $\nu$ is in $\mathbf{Z}^{n}$. Equations (9) and (11) imply

$$
\exp Q_{l}\left(\begin{array}{c}
\vartheta(t) \\
0 \\
\vdots \\
0
\end{array}\right)=\left(\begin{array}{c}
\vartheta(t) \\
0 \\
\vdots \\
0
\end{array}\right)
$$

for $1 \leq l \leq n$. This proves (8).

To verify (7) we note the following relations:

$$
\frac{\partial}{\partial \nu_{l}} e_{\nu}(t)=P_{l} e_{\nu}(t) \quad \text { for } 1 \leq l \leq n
$$

(Cf. Berenstein-Kawai-Struppa-Takei [3]). Hence we find

$$
\exp P_{l} e_{\nu}(t)=e_{\nu(l)}(t)
$$

for each $\nu$ in $\mathbf{R}^{n}$ and $1 \leq l \leq n$, where $\nu(l)$ denotes $\left(\nu_{1}+\delta_{l 1}, \ldots, \nu_{n}+\delta_{l n}\right)^{\prime}$. Therefore (9) and (12) give

$$
\exp P_{l}\left(\begin{array}{c}
\vartheta(t) \\
0 \\
\vdots \\
0
\end{array}\right)=\left(\begin{array}{c}
\vartheta(t) \\
0 \\
\vdots \\
0
\end{array}\right)
$$

This proves (7).

Q.E. D.

Thus we have obtained the simultaneous equations which the thetazerovalue satisfies. However the simultaneous equations (7) and (8) are not a well-defined system as they stand; to construct a well-defined system we introduce auxiliary operators $R_{[l, m]}$ and $S_{[k, l, m]}(1 \leq k, l, m \leq n)$ in the following manner: 


$$
\begin{aligned}
R_{[l, m]} & =\left[Q_{l}, Q_{m}\right], \\
S_{[k, l, m]} & =\left[Q_{k}, R_{[l, m]}\right] .
\end{aligned}
$$

Here and in what follows $[P, Q]$ denotes the commutator of operators $P$ and $Q$; $[P, Q]=P Q-Q P$. By a simple computation the explicit forms of the operators $R_{[l, m]}, S_{[k, l, m]}(1 \leq k, l, m \leq n)$ are given as follows:

$$
R_{[l, m]}=2 \pi \sqrt{-1}\left(\begin{array}{ccccccccccc} 
& \multicolumn{1}{c}{(l+1) \text {-th }} & \multicolumn{1}{c}{{ }^{(m+1)-\text { th }}} & & \\
0 & \cdots & 0 & 0 & 0 & \cdots & 0 & 0 & 0 & \cdots & 0 \\
0 & \cdots & 0 & -\partial_{m 1} & 0 & \cdots & 0 & \partial_{l 1} & 0 & \cdots & 0 \\
\vdots & & \vdots & \vdots & \vdots & & \vdots & \vdots & \vdots & & \vdots \\
0 & \cdots & 0 & -\partial_{m j} & 0 & \cdots & 0 & \partial_{l j} & 0 & \cdots & 0 \\
\vdots & & \vdots & \vdots & \vdots & & \vdots & \vdots & \vdots & & \vdots \\
0 & \cdots & 0 & -\partial_{m n} & 0 & \cdots & 0 & \partial_{l n} & 0 & \cdots & 0
\end{array}\right) \text {, }
$$

(14) $S_{[k, l, m]}=2 \pi \sqrt{-1} \times$

$$
\left(\begin{array}{cccc}
0 & \delta_{m 1} \partial_{k l}-\delta_{l 1} \partial_{k m} & \cdots & \delta_{m n} \partial_{k l}-\delta_{l n} \partial_{k m} \\
2 \pi \sqrt{-1}\left(\partial_{k l} \partial_{m 1}-\partial_{k m} \partial_{l 1}\right) & 0 & \cdots & 0 \\
\vdots & \vdots & & \vdots \\
2 \pi \sqrt{-1}\left(\partial_{k l} \partial_{m j}-\partial_{k m} \partial_{l j}\right) & 0 & \cdots & 0 \\
\vdots & \vdots & & \vdots \\
2 \pi \sqrt{-1}\left(\partial_{k l} \partial_{m n}-\partial_{k m} \partial_{l n}\right) & 0 & \cdots & 0
\end{array}\right) .
$$

Here the symbol $(l+1)$-th indicates the $(l+1)$-th column of the matrices. The importance of the operators introduced above is manifest by the following theorem.

Theorem 2.2. The operators $P_{l}, Q_{m}, R_{[l, m]}$ and $S_{[k, l, m]}$ satisfy the following commutation relations:

$$
\begin{aligned}
{\left[P_{l}, P_{m}\right] } & =\sum_{j, k=1}^{n} t_{l k} t_{m j} R_{[k, j]}, \\
{\left[P_{l}, Q_{m}\right] } & =-2 \pi \sqrt{-1} \delta_{l m} I+\sum_{k=1}^{n} t_{l k} R_{[k, m]}, \\
{\left[P_{k}, R_{[l, m]}\right] } & =\sum_{j=1}^{n} t_{k j} S_{[j, l, m]}, \\
{\left[P_{\alpha}, S_{[\beta, l, m]}\right] } & =2 \pi \sqrt{-1} \partial_{\beta l}\left(\sum_{j=1}^{n} t_{\alpha j} R_{[j, m]}\right)-2 \pi \sqrt{-1} \partial_{\beta m}\left(\sum_{j=1}^{n} t_{\alpha j} R_{[j, l]}\right)
\end{aligned}
$$




$$
\begin{aligned}
& -2 \pi \sqrt{-1} \delta_{\alpha \beta} R_{[l, m]}-2 \pi \sqrt{-1} \delta_{\alpha l} \sum_{j=1}^{n} E_{j+1, \beta+1} R_{[j, m]} \\
& +2 \pi \sqrt{-1} \delta_{\alpha m} \sum_{j=1}^{n} E_{j+1, \beta+1} R_{[j, l]},
\end{aligned}
$$

$$
\left[Q_{\alpha}, S_{[\beta, l, m]}\right]=2 \pi \sqrt{-1} \partial_{\beta l} R_{[\alpha, m]}-2 \pi \sqrt{-1} \partial_{\beta m} R_{[\alpha, l]} .
$$

Here $E_{i j}$ denotes the $(i, j)$-matrix unit; $E_{i j}=\left(\delta_{i k} \delta_{j l}\right)_{1 \leq k, l \leq n+1}$.

Proof. Let us begin our discussion by verifying (16). As it follows from the definitions of $P_{l}$ and $Q_{k}$ that

$$
P_{l}=2 \pi \sqrt{-1} E_{l+1,1}+\sum_{k=1}^{n} t_{l k} Q_{k}
$$

holds, we find

$$
\left[P_{l}, Q_{m}\right]=2 \pi \sqrt{-1}\left[E_{l+1,1}, Q_{m}\right]+\sum_{k=1}^{n} t_{l k}\left[Q_{k}, Q_{m}\right]-\sum_{k=1}^{n}\left[Q_{m}, t_{l k} I\right] Q_{k} .
$$

On the other hand, a direct calculation shows

$$
\begin{aligned}
{\left[E_{l+1,1}, Q_{m}\right] } & =-\delta_{l m} \mathrm{E}_{11}+E_{l+1, m+1}, \\
{\left[Q_{m}, t_{l k} I\right] } & =2 \pi \sqrt{-1} \delta_{l m}\left(1+\delta_{k m}\right) E_{k+1,1}+2 \pi \sqrt{-1} \delta_{k m}\left(1-\delta_{l m}\right) E_{l+1,1} .
\end{aligned}
$$

Hence we have

$$
\begin{aligned}
{\left[P_{l}, Q_{m}\right]=} & -2 \pi \sqrt{-1} \delta_{l m} E_{11}+2 \pi \sqrt{-1} E_{l+1, m+1}+\sum_{k=1}^{n} t_{l k} R_{[k, m]} \\
& -2 \pi \sqrt{-1} \delta_{l m} \sum_{k=1}^{n}\left(1+\delta_{k m}\right) E_{k+1,1} Q_{k}-2 \pi \sqrt{-1}\left(1-\delta_{l m}\right) E_{l+1,1} Q_{m} \\
= & 2 \pi \sqrt{-1}\left\{-\delta_{l m} E_{11}+E_{l+1, m+1}-\delta_{l m} \sum_{k=1}^{n}\left(1+\delta_{k m}\right) E_{k+1, k+1}\right. \\
& \left.-\left(1-\delta_{l m}\right) E_{l+1, m+1}\right\}+\sum_{k=1}^{n} t_{l k} R_{[k, m]} \\
= & -2 \pi \sqrt{-1} \delta_{l m} I+\sum_{k=1}^{n} t_{l k} R_{[k, m]} .
\end{aligned}
$$

This proves (16).

Let us prove next (15). Using (20) again, we obtain

$$
\left[P_{l}, P_{m}\right]=2 \pi \sqrt{-1}\left[E_{l+1,1}, P_{m}\right]+\sum_{k=1}^{n} t_{l k}\left[Q_{k}, P_{m}\right]-\sum_{k=1}^{n}\left[P_{m}, t_{l k} I\right] Q_{k}
$$


On the other hand we find

$$
\left[P_{m}, t_{l k} I\right]=2 \pi \sqrt{-1} t_{l m} E_{k+1,1}+2 \pi \sqrt{-1} t_{k m} E_{l+1,1} .
$$

Hence we deduce from (21)

$$
\begin{aligned}
{\left[P_{l}, P_{m}\right]=} & -2 \pi \sqrt{-1} t_{l m} E_{11}+2 \pi \sqrt{-1} \sum_{k=1}^{n} t_{k m} E_{l+1, k+1}-\sum_{j, k=1}^{n} t_{l k} t_{m j} R_{[j, k]} \\
& +2 \pi \sqrt{-1} t_{l m} I-2 \pi \sqrt{-1} \sum_{k=1}^{n}\left(t_{l m} E_{k+1, k+1}+t_{k m} E_{l+1, k+1}\right) \\
= & \sum_{j, k=1}^{n} t_{l k} t_{m j} R_{[j, k]} .
\end{aligned}
$$

This proves (15).

The verification of (17) is again based upon (20); the relation (20) implies

$$
\left[P_{k}, R_{[l, m]}\right]=2 \pi \sqrt{-1}\left[E_{k+1,1}, R_{[l, m]}\right]+\sum_{j=1}^{n} t_{k j}\left[Q_{j}, R_{[l, m]}\right]-\sum_{j=1}^{n}\left[R_{[l, m]}, t_{k j} I\right] Q_{j}
$$

On the other hand, a direct computation shows

$$
\left[E_{k+1,1}, R_{[l, m]}\right]=2 \pi \sqrt{-1} \sum_{j=1}^{n}\left(\delta_{k l} \partial_{j m}-\delta_{k m} \partial_{j l}\right) E_{j+1,1}
$$

Hence (22) entails

$$
\begin{aligned}
{\left[P_{k}, R_{[l, m]}\right]=} & (2 \pi \sqrt{-1})^{2} \sum_{j=1}^{n}\left(\delta_{k l} \partial_{j m}-\delta_{k m} \partial_{j l}\right) E_{j+1,1}+\sum_{j=1}^{n} t_{k j} S_{[j, l, m]}-2 \pi \sqrt{-1} \\
& \times\left\{\sum_{j=1}^{n}\left(\delta_{k l} E_{j+1, m+1}-\delta_{k m} E_{j+1, l+1}\right) Q_{j}+E_{k+1, m+1} Q_{l}-E_{k+1, l+1} Q_{m}\right\} \\
= & (2 \pi \sqrt{-1})^{2}\left\{\sum_{j=1}^{n}\left(\delta_{k l} \partial_{j m}-\delta_{k m} \partial_{j l}\right) E_{j+1,1}-\sum_{j=1}^{n}\left(\delta_{k l} \partial_{j m}-\delta_{k m} \partial_{j l}\right) E_{j+1,1}\right. \\
& \left.-\partial_{l m} E_{k+1,1}+\partial_{l m} E_{k+1,1}\right\}+\sum_{j=1}^{n} t_{j k} S_{[j, l, m]} \\
= & \sum_{j=1}^{n} t_{j k} S_{[j, l, m]} .
\end{aligned}
$$

This proves (17).

To prove (18), we use (14); it immediately follows from (14) and the definition of $Q_{m}$ that

$$
S_{[\beta, l, m]}=2 \pi \sqrt{-1}\left(\partial_{\beta l} Q_{m}-\partial_{\beta m} Q_{l}\right)
$$


holds for $1 \leq \beta, l, m \leq n$. On the other hand, we can easily verify the following commutation relations:

$$
\left[P_{l}, \partial_{i j} I\right]=-\delta_{i l} Q_{j}-\delta_{j l} Q_{i}
$$

for $1 \leq i, j, l \leq n$. Equations (16), (23) and (24) entail

$$
\begin{aligned}
{\left[P_{\alpha}, S_{[\beta, l, m]}\right]=} & 2 \pi \sqrt{-1}\left[P_{\alpha}, \partial_{\beta l} Q_{m}\right]-2 \pi \sqrt{-1}\left[P_{\alpha}, \partial_{\beta m} Q_{l}\right] \\
= & 2 \pi \sqrt{-1}\left(\partial_{\beta l}\left[P_{\alpha}, Q_{m}\right]+\left[P_{\alpha}, \partial_{\beta l} I\right] Q_{m}-\partial_{\beta m}\left[P_{\alpha}, Q_{l}\right]\right. \\
& \left.-\left[P_{\alpha}, \partial_{\beta m} I\right] Q_{l}\right) \\
= & (2 \pi \sqrt{-1})^{2}\left(\delta_{\alpha l} \partial_{\beta m} I-\delta_{\alpha m} \partial_{\beta l} I\right)+2 \pi \sqrt{-1}\left\{\partial_{\beta l}\left(\sum_{j=1}^{n} t_{\alpha j} R_{[j, m]}\right)\right. \\
& -\partial_{\beta m}\left(\sum_{j=1}^{n} t_{\alpha j} R_{[j, l]}\right)-\delta_{\alpha \beta} Q_{l} Q_{m}-\delta_{\alpha l} Q_{\beta} Q_{m}+\delta_{\alpha \beta} Q_{m} Q_{l} \\
& \left.+\delta_{\alpha m} Q_{\beta} Q_{l}\right\} .
\end{aligned}
$$

Further, a straightforward computation shows

$$
\begin{aligned}
& -Q_{\beta} Q_{m}+2 \pi \sqrt{-1} \partial_{\beta m} I=2 \pi \sqrt{-1} \times \\
& \left(\begin{array}{cccccccc}
\multicolumn{10}{c}{(m+1) \text {-th }} & & & \\
0 & 0 & \cdots & 0 & 0 & 0 & \cdots & 0 \\
0 & \partial_{\beta m} & & 0 & -\partial_{\beta 1} & 0 & \cdots & 0 \\
\vdots & & \ddots & & \vdots & \vdots & & \vdots \\
0 & & & \partial_{\beta m} & -\partial_{\beta, m-1} & 0 & \cdots & 0 \\
0 & & \cdots & 0 & 0 & 0 & \cdots & 0 \\
0 & & \cdots & 0 & -\partial_{\beta, m+1} & \partial_{\beta m} & & 0 \\
\vdots & & & \vdots & \vdots & & \ddots & \\
0 & 0 & \cdots & 0 & -\partial_{\beta n} & 0 & & \partial_{\beta m}
\end{array}\right) .
\end{aligned}
$$

Hence we find

$$
-Q_{\beta} Q_{m}+2 \pi \sqrt{-1} \partial_{\beta m} I=-\sum_{j=1}^{n} E_{j+1, \beta+1} R_{[j, m]}
$$

Using (26), we deduce from (25)

$$
\begin{aligned}
{\left[P_{\alpha}, S_{[\beta, l, m]}\right]=} & 2 \pi \sqrt{-1}\left\{\partial_{\beta l}\left(\sum_{j=1}^{n} t_{\alpha j} R_{[j, m]}\right)-\partial_{\beta m}\left(\sum_{j=1}^{n} t_{\alpha j} R_{[j, t]}\right)-\delta_{\alpha \beta} R_{[l, m]}\right\} \\
& -2 \pi \sqrt{-1}\left(\delta_{\alpha l} \sum_{j=1}^{n} E_{j+1, \beta+1} R_{[j, m]}-\delta_{\alpha m} \sum_{j=1}^{n} E_{j+1, \beta+1} R_{[j, l]}\right) .
\end{aligned}
$$


This proves (18).

Finally the relation (19) immediately follows from (23) as $Q_{\alpha}$ and $\partial_{\beta l}$ commute;

$$
\begin{aligned}
{\left[Q_{\alpha}, S_{[\beta, l, m]}\right] } & =2 \pi \sqrt{-1}\left[Q_{\alpha}, \partial_{\beta l} Q_{m}\right]-2 \pi \sqrt{-1}\left[Q_{\alpha}, \partial_{\beta m} Q_{l}\right] \\
& =2 \pi \sqrt{-1} \partial_{\beta l}\left[Q_{\alpha}, Q_{m}\right]-2 \pi \sqrt{-1} \partial_{\beta m}\left[Q_{\alpha}, Q_{l}\right] \\
& =2 \pi \sqrt{-1} \partial_{\beta l} R_{[\alpha, m]}-2 \pi \sqrt{-1} \partial_{\beta m} R_{[\alpha, l]} .
\end{aligned}
$$

This completes the proof of Theorem 2.2.

Q.E. D.

If we let $\mathcal{N}$ denote the coherent left $\mathscr{D}_{X}$-module

$$
\mathscr{D}_{X}^{n+1} /\left(\sum_{1 \leq l, m \leq n} \mathscr{D}_{X}^{n+1} R_{[l, m]}+\sum_{1 \leq k, l, m \leq n} \mathscr{D}_{X}^{n+1} S_{[k, l, m]}\right)
$$

Theorem 2.2 guarantees that $P_{l}$ and $Q_{m}$ act on $\mathcal{N}$ from the right and that they satisfy the following commutation relations in $\mathscr{E} n d_{\mathscr{D}_{X}}(\mathcal{N})$ :

$$
\begin{aligned}
& {\left[P_{l}, P_{m}\right]=0,} \\
& {\left[P_{l}, Q_{m}\right]=-2 \pi \sqrt{-1} \delta_{l m} I,} \\
& {\left[Q_{l}, Q_{m}\right]=0 .}
\end{aligned}
$$

It is also clear from the definitions of $R_{[l, m]}$ and $S_{[k, l, m]}$ together with (10) that $R_{[k, l]}$ and $S_{[k, l, m]}$ annihilate $e_{\nu}(t)$ and hence $(\vartheta(t), 0, \ldots, 0)^{\prime}$. Furthermore we can readily verify ord $P_{l}$ and ord $Q_{m}$ are strictly smaller than 1. (Cf. [8]. See also the choice of $\rho$ in the proof of Proposition 2.5 below.) Hence $\Phi_{l}=\exp P_{l}-I$ and $\Psi_{m}=\exp Q_{m}-I$ are well-defined differential operators of infinite order. It also follows from the relations (27), (28) and (29) and Lemma 1.2 in [8] that $\Phi_{l}$ 's and $\Psi_{m}$ 's do commute in the endomorphism ring of $\mathscr{D}_{X}^{\infty} \otimes_{\mathscr{D}_{X}} \mathcal{N}, \mathscr{E}_{n} d_{\mathscr{D}_{X}^{\infty}}\left(\mathscr{D}_{X}^{\infty} \otimes\right.$ $\left.\mathscr{D}_{x} \mathcal{N}\right)$. Thus we can introduce the following Koszul complex $K$ using $\Phi_{l}$ and $\Psi_{m}$.

Definition 2.3. Let $\mathcal{N}^{\infty}$ denote $\mathscr{D}_{X}^{\infty} \otimes \mathscr{D}_{X} \mathcal{N}$ and let $K$ denote the following Koszul complex:

$$
0 \leftarrow \mathcal{N}^{\infty} \stackrel{\Phi_{1,} \quad . \Phi_{n}, \Psi_{1,} \quad . \Psi_{n}}{\longleftarrow}\left(\mathcal{N}^{\infty}\right)^{2 n} \longleftarrow \cdots \leftarrow\left(\mathcal{N}^{\infty}\right)^{2 n} \longleftarrow \mathcal{N}^{\infty} \leftarrow 0
$$

As is shown in [4], $K$ is a good complex in the sense of [9], and hence we can discuss its characteristic set $\mathrm{Ch}(K)$. The rest of this section is devoted to the concrete description of $\mathrm{Ch}(K)$. In what follows we use the fiber coordinate 
$\tau_{i j}$ of $T^{*} X$ given by $\tau_{i j}=\sigma_{1}\left(\partial_{i j}\right)$, where $\sigma_{1}\left(\partial_{i j}\right)$ is the principal symbol of order 1 of $\partial_{i j}$. We denote the matrix $\left(\tau_{i j}\right)_{1 \leq i, j \leq n}$ by $\tau$. Our discussion is based on the following lemma, which is a variant of Aoki [1] (cf. [2] also) concering the invertibility of microdifferential operators.

Lemma 2.4. ([4]) Let $A=\left(A_{i j}\right)_{1 \leq i, j \leq n}$ be an $n \times n$ matrix of microdifferential operators of finite order. Let $\left\{r_{i}\right\}_{1 \leq i \leq n}$ be a set of rational numbers and let $\rho$ be a real number strictly smaller than 1 . Let us consider the following conditions $(a)$ and (b):

(a) $\quad \operatorname{ord} A_{i j} \leq r_{i}-r_{j}+\rho$ for $1 \leq i, j \leq n$

(b) every eigenvalue of the matrix $\left(\sigma_{r_{t}-r,+\rho}\left(A_{\imath j}\left(x^{*}\right)\right)\right)_{1 \leq i, j \leq n}$ is contained in $\mathbf{C} \backslash \sqrt{-1} \mathbf{R}$,

where $\sigma_{\lambda}\left(A_{i j}\right)$ denotes the principal symbol of $A_{i j}$ and $\sigma_{\lambda}\left(P_{i j}\right)\left(x^{*}\right)$ denotes its value at a point $x^{*}$ of the cotangent bundle $T^{*} X$. Then under the condition $(a), \exp A$ is a well-defined section of the sheaf of $n \times n$ matrices of holomorphic microlocal operators $M\left(n \times n ; \mathscr{E}_{X}^{R}\right)$. If the condition $(b)$ is further satisfied, $\exp A-I$ is invertible in $M$ $\left(n \times n ; \mathscr{E}_{X, x *}^{R}\right)$.

If one of the operators $\Phi_{l}$ and $\Psi_{m}$ is invertible, then the complex $K$ is exact. Hence this lemma gives a sharp result on the description of the characteristic set of $K$ (Proposition 2.5 below). We hope to make use of the result in future to study the structure of the cohomology groups $\mathscr{H}^{j}\left(\mathbf{R} \operatorname{Hom}_{\mathscr{D}_{X}^{\mathfrak{x}}}\left(K, \mathscr{O}_{X}\right)\right)$; in view of the result of [4] and Proposition 2.5 below, we conjecture that, if the signature of $\Im t$ is $(n-q, q)$, then

$$
\mathscr{H}^{j}\left(\mathbf{R} \operatorname{Hom}_{\mathscr{D}_{X}^{\infty}}\left(K, \mathscr{O}_{X}\right)\right)_{t}= \begin{cases}0 & (j \neq q) \\ \mathbf{C} & (j=q) .\end{cases}
$$

Here and in what follows the real part (resp., imaginary part) of a complex matrix $A$ denotes $\Re A$ (resp., $\Im A$ ).

Proposition 2.5. Let $(t ; \tau)$ in $T^{*} X$ be a point in $\mathrm{Ch}(K)$, the characteristic set of the Koszul complex $K$ given in Definition 2.3. Then it satisfies the following conditions (2.5.1), (2.5.2), (2.5.3) and (2.5.4):

(2.5.1) the determinant of each $2 \times 2$ minor matrix of $\tau$ vanishes:

$$
\operatorname{det}\left(\begin{array}{cc}
\tau_{l j} & \tau_{l k} \\
\tau_{m j} & \tau_{m k}
\end{array}\right)=0 \quad \text { for } \quad 1 \leq l<m \leq n \text { and } 1 \leq j<k \leq n
$$


(2.5.2) the real part of $\tau$ is equal to 0 :

$$
\Re \tau=0,
$$

(2.5.3) the imaginary part of each diagonal element of $\tau$ is nonnegative:

$$
\Im \tau_{l l} \geq 0 \quad \text { for } \quad 1 \leq l \leq n
$$

(2.5.4) the real part of the matrix $t \tau$ is zero:

$$
\Re t \tau=0
$$

Remark. The condition (2.5.1) shows that the rank of $\tau$ is less than or equal to 1 . The conditions $(2.5 .1),(2.5 .2)$ and (2.5.3) show that the imaginary part of each $j \times j(1 \leq j \leq n+1)$ principal minor matrix of $\tau$ is positive semidefinite.

Proof of Proposition 2.5. Let us consider the following differential equations:

$$
\begin{aligned}
& R_{[l, m]} u=0, \\
& S_{[k l, m]} u=0
\end{aligned}
$$

for $1 \leq k, l, m \leq n$. Here $u$ denotes an $(n+1)$-column vector $\left(u_{0}, \cdots, u_{n}\right)^{\prime}$. Using the explicit form of $R_{[l, m]}$, we deduce from (30)

$$
\partial_{m j} u_{l}-\partial_{l j} u_{m}=0
$$

for $1 \leq j, l, m \leq n$. Operating $\partial_{k \alpha}$ to both sides of (32), we have

$$
\partial_{m j} \partial_{k \alpha} u_{l}-\partial_{l j} \partial_{k \alpha} u_{m}=0
$$

for $1 \leq \alpha, j, k, l, m \leq n$. Since $\partial_{k \alpha} u_{l}=\partial_{k l} u_{\alpha}$ and $\partial_{k \alpha} u_{m}=\partial_{k m} u_{\alpha}$ hold by (32), (33) entails the following equations:

$$
\partial_{k l} \partial_{m j} u_{\alpha}-\partial_{k m} \partial_{l j} u_{\alpha}=0
$$

for $1 \leq \alpha, j, k, l, m \leq n$. On the other hand (31) gives

$$
\partial_{k l} \partial_{m j} u_{0}-\partial_{k m} \partial_{l j} u_{0}=0
$$

for $1 \leq j, k, l, m \leq n$. Therefore this proves that each point in $\mathrm{Ch}(K)$ should 
satisfy the condition (2.5.1).

Using (2.5.1), we next show that each point in $\mathrm{Ch}(K)$ satisfies the conditions (2.5.2) and (2.5.3). If $\tau=0$, a point $(t ; \tau)$ in $\mathrm{Ch}(K)$ trivially satisfies (2.5.2) and (2.5.3). Hence we may assume without loss of generality that $\tau_{i j}$ $\neq 0$ for some $i$ and $j$. We may further assume that $\tau_{i i} \neq 0$, because $\tau_{i j}^{2}=\tau_{i i} \tau_{j j}$ follows from (2.5.1) if $i \neq j$. We then renumber the indices so that $\tau_{11} \neq 0$ may hold. Since $\sqrt{2 \pi \sqrt{-1} \partial_{11}}-1$ is then well-defined on the points in question, let us introduce the following $(n+1) \times(n+1)$ matrix $\widetilde{Q}_{1}$ of microdifferential operators:

$$
\begin{aligned}
\widetilde{Q}_{1} & =Q_{1}-\frac{1}{\sqrt{2 \pi \sqrt{-1} \partial_{11}}} \sum_{k=2}^{n} E_{k+1,2} R_{[1, k]} \\
& =2 \pi \sqrt{-1} \times
\end{aligned}
$$

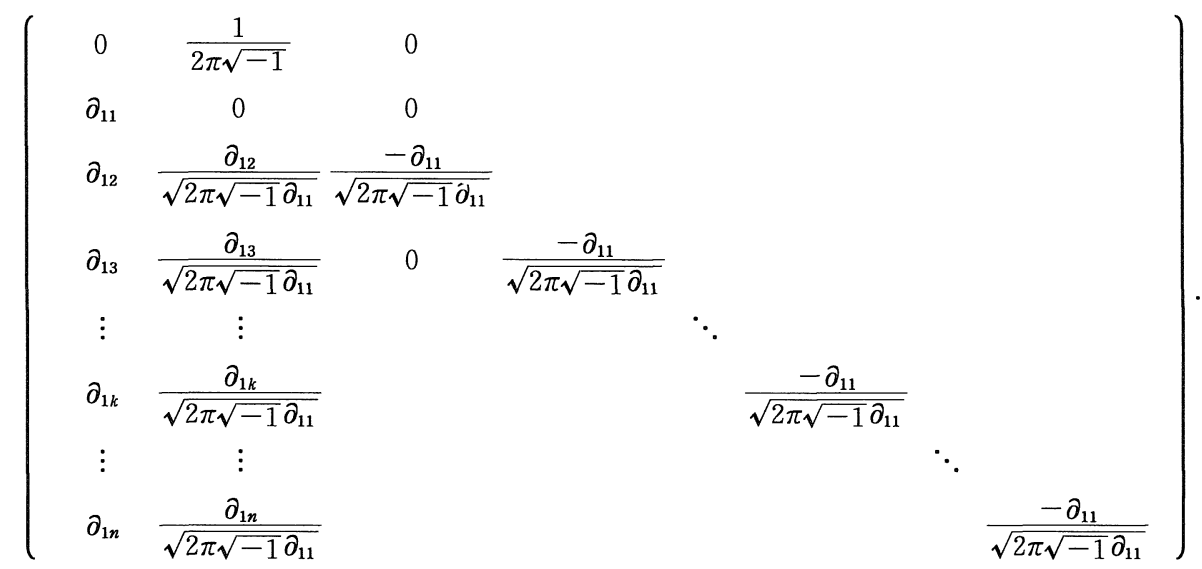

Note that $Q_{1}=\widetilde{Q}_{1}$ holds on $\mathscr{E} n d\left(\mathscr{E}_{X}^{\mathbb{R}} \otimes_{p^{-1} \mathscr{D}_{x}} p^{-1} \mathcal{N}\right)$ where $p$ denotes the projection from $T^{*} X$ to $X$. Hence it suffices to study the microdifferential operator $\widetilde{Q}_{1}$ instead of $Q_{1}$ for the description of $\mathrm{Ch}(K)$. We use Lemma 2.4 to prove that each point in $\mathrm{Ch}(K)$ satisfies the conditions $(2.5 .2)$ and $(2.5 .3)$. To employ Lemma 2.4 we set

$$
\begin{aligned}
& r_{i}=\left\{\begin{array}{ll}
\frac{1}{2} & \text { for } i=1 \\
1 & \text { for } 2 \leq i \leq \mathrm{n}+1
\end{array},\right. \\
& \rho=\frac{1}{2} .
\end{aligned}
$$

For simplicity we denote $\left(\sigma_{r_{i}-r,+\rho}\left(\widetilde{Q}_{1}\right)\right)_{1 \leq i, j \leq n+1}$ by $\sigma\left(\widetilde{Q}_{1}\right)(t ; \tau)$. Then the explicit form of $\sigma\left(\widetilde{Q}_{1}(t ; \tau)\right)$ is as follows: 
$\sigma\left(\widetilde{Q_{1}}\right)(t ; \tau)=2 \pi \sqrt{-1} \times$

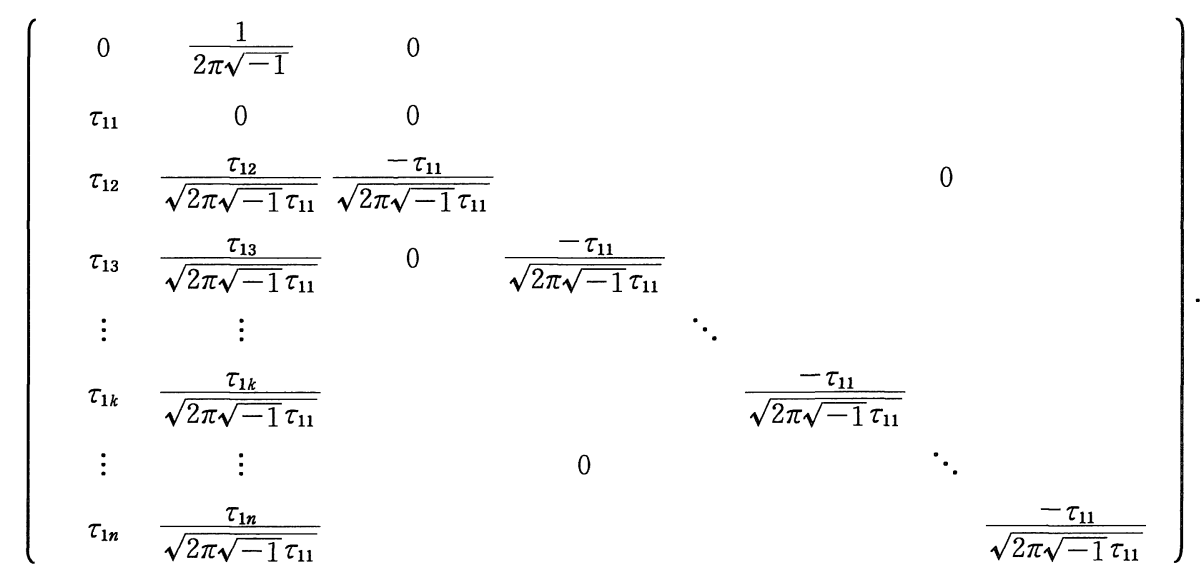

Hence its characteristic polynomial is

$$
\left(\lambda^{2}-2 \pi \sqrt{-1} \tau_{11}\right)\left(\lambda+\sqrt{2 \pi \sqrt{-1} \tau_{11}}\right)^{n-1} .
$$

Thus we find that the eigenvalues of $\sigma\left(\widetilde{Q}_{1}\right)(t ; \tau)$ are $\pm \sqrt{2 \pi \sqrt{-1} \tau_{11}}$. Hence Lemma 2.4 asserts that $\mathrm{Ch}(K)$ is contained in

$$
\left\{(t ; \tau) \in T^{*} X \mid \tau_{11} \in \sqrt{-1} \mathbf{R}_{>0}\right\}
$$

where $\mathbb{R}_{>0}$ denotes the set of all strictly positive real numbers. Letting $\mathbb{R}_{\geq 0}$ denote the set $\mathbb{R}_{>0} \cup\{0\}$, we further find

$$
\mathrm{Ch}(K) \subset\left\{(t ; \tau) \in T^{*} X \mid \tau_{l l} \in \sqrt{-1} \mathbf{R}_{\geq 0} \quad \text { for } \quad 1 \leq l \leq n\right\},
$$

which proves that each point in $\mathrm{Ch}(K)$ satisfies $(2.5 .3)$. In fact, if $(t ; \tau)$ is in $\mathrm{Ch}(K)$ and $\tau_{l l}=0$, (35) is trivial. If $\tau_{l l} \neq 0$, by the same reasoning as above we find $\tau_{l l} \in \sqrt{-1} \mathbf{R}_{>0}$ by replacing $Q_{1}$ with $Q_{l}$. Thus we obtain (35). The condition (2.5.1) and (35) shows that $\tau^{2}{ }_{l m}=\tau_{l l} \tau_{m m} \in \mathbb{R}_{\leq 0}$. Hence we have $\tau_{l m} \in \sqrt{-1} \mathbf{R}$ for $1 \leq l, m \leq n$. This proves that each point of $\mathrm{Ch}(K)$ satisfies the condition (2.5.2).

Finally let us prove that each point of $\mathrm{Ch}(K)$ satisfies the condition (2.5.4). If $\tau=0$, there is nothing to prove. Hence without loss of generality we may assume that $\tau_{i j} \neq 0$ for some $i$ and $j$. Furthermore for a point $(t ; \tau)$ in $\mathrm{Ch}(K)$ we may assume that $\tau_{i i} \neq 0$ by $(2.5 .1)$. Renumbering the indices, we suppose that $\tau_{11} \neq 0$. In parallel with $Q_{1}$ we introduce the following $(n+1) \times(n$ $+1)$ matrices $\widetilde{P}_{l}(l=1, \cdots, n)$ of microdifferential operators with parameters; 


$$
\widetilde{P}_{l}=P_{l}-\frac{1}{\sqrt{2 \pi \sqrt{-1} \partial_{11}}} \sum_{1 \leq \alpha<\beta \leq n} X(\alpha, \beta) R_{[\alpha, \beta]}
$$

where $X(\alpha, \beta)$ denotes an $(n+1) \times(n+1)$ matrix of parameters of the following form:

$$
X(\alpha, \beta)=\left(\begin{array}{cccccc}
0 & 0 & \cdots & 0 & \cdots & 0 \\
0 & X(\alpha, \beta)_{11} & \cdots & X(\alpha, \beta)_{1 n} & \cdots & X(\alpha, \beta)_{1 n} \\
\vdots & \vdots & & \vdots & & \vdots \\
0 & X(\alpha, \beta)_{g 1} & \cdots & X(\alpha, \beta)_{g h} & \cdots & X(\alpha, \beta)_{g n} \\
\vdots & \vdots & & \vdots & & \vdots \\
0 & X(\alpha, \beta)_{n 1} & \cdots & X(\alpha, \beta)_{n h} & \cdots & X(\alpha, \beta)_{n n}
\end{array}\right)
$$

for $1 \leq \alpha<\beta \leq n$ with $X(\alpha, \beta)_{g h}(1 \leq g, h \leq n)$ being complex numbers. As is the same with $\widetilde{Q}_{1}$, the operator $\widetilde{P}_{l}$ is well-defined and gives rise to the same element as $P_{l}$ in $\mathscr{E}_{n d}\left(\mathscr{E}_{X}^{\mathbf{R}} \otimes_{p^{-1} \mathscr{D}_{X}} p^{-1} \mathcal{N}\right)$. To discuss the invertibility of $\exp \widetilde{P}_{l}-I$ we again employ Lemma 2,4 with choosing $r_{i}$ and $\rho$ as follows:

$$
\begin{aligned}
& r_{i}=\left\{\begin{array}{ll}
\frac{1}{2} & \text { for } \quad i=1 \\
1 & \text { for } \quad 2 \leq i \leq \mathrm{n}+1
\end{array},\right. \\
& \rho=\frac{1}{2} \text {. }
\end{aligned}
$$

In what follows we dnote $\sigma_{r_{1}-r,+\rho}\left(\widetilde{P}_{l}\right)_{1 \leq l, j \leq n}$ by $\sigma\left(\widetilde{P}_{l}\right)(t ; \tau)$ for the simplicity of notations. By a straightforward calculation we can easily verify that each entry of the matrix $\sigma\left(\widetilde{P}_{l}\right)(t ; \tau)$ is as follows:

$$
\begin{array}{rlrl}
\sigma\left(\widetilde{P}_{l}\right)(t ; \tau)_{g+1, h+1} & =0 & & \text { if } \quad g=0, h=0, \\
& =t_{h l} & & \text { if } \quad g=0,1 \leq h \leq n, \\
& =2 \pi \sqrt{-1} \sum_{k=1}^{n} t_{l k} \tau_{k g} & & \text { if } \quad 1 \leq g \leq n, h=0, \\
& =\frac{2 \pi \sqrt{-1}}{\sqrt{2 \pi \sqrt{-1} \tau_{11}} \sum_{k=1}^{n} \sum_{m=2}^{n} X(1, m)_{g k} \tau_{m k}} \quad \text { if } \quad 1 \leq g \leq n, h=1, \\
& =-\frac{2 \pi \sqrt{-1}}{\sqrt{2 \pi \sqrt{-1} \tau_{11}} \sum_{k=1}^{n} \sum_{m=1}^{n-1} X(m, n)_{g k} \tau_{m k}} \quad & & \text { if } \quad 1 \leq g \leq n, h=n, \\
& =\frac{2 \pi \sqrt{-1}}{\sqrt{2 \pi \sqrt{-1} \tau_{11}} \sum_{k=1}^{n}\left(\sum_{m=n+1}^{n} X(h, m)_{g k} \tau_{m k}\right.} &
\end{array}
$$




$$
\left.-\sum_{m=1}^{h-1} X(m, h)_{g k} \tau_{m k}\right) \quad \text { if } \quad 1 \leq g \leq n, 2 \leq h \leq n-1
$$

We now prepare some lemmas concerning the structure of the eigenvalues of $\sigma\left(\tilde{P}_{l}\right)(t ; \tau)$ which do not depend on $\{X(\alpha, \beta)\}_{1 \leq \alpha, \beta \leq n, \alpha<\beta}$; as we shall show later, the eigenvalues relevant to $\mathrm{Ch}(K)$ are those independent of $X(\alpha, \beta)$ and it is why we prepare the following lemmas.

Lemma 2.6. If a point $(t ; \tau)$ in $T^{*} X$ satisfies the condition $(2.5 .1)$, then the eigenvalues of $\sigma\left(\widetilde{P}_{l}\right)(t ; \tau)$ which do not depend on $\{X(\alpha, \beta)\}_{1 \leq \alpha, \beta \leq n, \alpha<\beta}$ are either 0 or $\pm \frac{2 \pi \sqrt{-1}}{\sqrt{2 \pi \sqrt{-1} \tau_{11}}} \sum_{k=1}^{n} t_{l k} \tau_{k 1}$. If $(t ; \tau)$ in $T^{*} X$ further satisfies $\sum_{k=1}^{n} t_{l k} \tau_{k 1}$ $\neq 0$, then the eigenvalues of $\sigma\left(\tilde{P}_{l}\right)(t ; \tau)$ which are independent of $\{X(\alpha, \beta)\}_{1 \leq \alpha, \beta \leq n, \alpha<\beta}$ are at most $\pm \frac{2 \pi \sqrt{-1}}{\sqrt{2 \pi \sqrt{-1} \tau_{11}}} \sum_{k=1}^{n} t_{l k} \tau_{k 1}$.

Proof. If an eigenvalue $\lambda$ of $\sigma\left(\widetilde{P}_{l}\right)(t ; \tau)$ does not depend on $X(\alpha, \beta) \quad(1 \leq \alpha$ $<\beta \leq n)$, then $\lambda$ is also an eigenvalue of $\sigma\left(\widetilde{P}_{l}\right)(t ; \tau)$ at $X(\alpha, \beta)=0$ for $1 \leq \alpha<\beta$ $\leq n$. This means that $\lambda$ is an eigenvalue of $\sigma\left(P_{l}\right)(t ; \tau)$. Suppose that $\lambda \neq 0$ and denote its non-zero eigenvector by $\phi=\left(\phi_{0}, \cdots, \psi_{n}\right)^{\prime}$. Writing down the relations in a componentwise, we have

$$
\begin{gathered}
\sum_{k=1}^{n} t_{l k} \phi_{k}=\lambda \phi_{0}, \\
2 \pi \sqrt{-1} \sum_{k=1}^{n} t_{l k} \tau_{k j} \phi_{0}=\lambda \phi_{j}
\end{gathered}
$$

for $1 \leq j \leq n$. Relations (36) and (37) entail

$$
\lambda^{2}=2 \pi \sqrt{-1} \sum_{j, k=1}^{n} t_{l j} t_{l k} \tau_{k j}
$$

On the other hand the condition (2.5.1) gives $\tau_{k j}=\tau_{11}^{-1} \tau_{k 1} \tau_{j 1}$ for $1 \leq j, k \leq n$.

Hence we have

$$
\begin{aligned}
\lambda^{2} & =\frac{2 \pi \sqrt{-1}}{\tau_{11}} \sum_{j, k=1}^{n} t_{l j} \tau_{j 1} t_{l k} \tau_{k 1} \\
& =\frac{2 \pi \sqrt{-1}}{\tau_{11}}\left(\sum_{k=1}^{n} t_{l k} \tau_{k 1}\right)^{2}
\end{aligned}
$$


This proves that the eigenvalues of $\sigma\left(\widetilde{P}_{l}\right)(t ; \tau)$ which do not depend on $\{X(\alpha$, $\beta)\}_{1 \leq \alpha, \beta \leq n, \alpha<\beta}$ are either 0 or $\pm \frac{2 \pi \sqrt{-1}}{\sqrt{2 \pi \sqrt{-1} \tau_{11}}} \sum_{k=1}^{n} t_{l k} \tau_{k l}$.

Suppose that 0 is an eigenvalue of $\sigma\left(\widetilde{P}_{l}\right)(t ; \tau)$ which is independent of $\{X(\alpha, \beta)\}_{1 \leq \alpha, \beta \leq n, \alpha<\beta}$. Then we have

$$
\operatorname{det} \sigma\left(\widetilde{P}_{l}\right)(t ; \tau)=0
$$

for arbitrary $X(\alpha, \beta)$. We can regard the determinant of $\sigma\left(\widetilde{P}_{l}\right)(t ; \tau)$ as a polynomial of $X(\alpha, \beta)_{g h}(1 \leq \alpha<\beta \leq n, 1 \leq g, h \leq n)$. Let a set $Z$ in $T^{*} X$ be the totality of the common zeros of all coefficients of the determinant of $\sigma\left(\widetilde{P}_{l}\right)(t ; \tau)$ as a polynomial of $X(\alpha, \beta)_{g h}(1 \leq \alpha<\beta \leq n, 1 \leq g, h \leq n)$. Using the explicit form of $\sigma\left(\widetilde{P_{l}}\right)(t ; \tau)$ we find that the coefficient of $\Pi_{\beta=2}^{n} X(1, \beta)_{\beta 1}$ is given as follows:

$$
(-1)^{n}(\sqrt{2 \pi \sqrt{-1}})^{\frac{n+1}{2}} \tau_{11} \frac{n-3}{2}\left(\sum_{k=1}^{n} t_{l k} \tau_{k 1}\right)^{2}
$$

Therefore we have

$$
\left.Z \subset\{t ; \tau) \in T^{*} X \mid \sum_{k=1}^{n} t_{l k} \tau_{k 1}=0\right\}
$$

Hence if $\sum_{k=1}^{n} t_{l k} \tau_{k l} \neq 0,(t ; \tau)$ does not belong to to the set $Z$. Then there exist $X(\alpha, \beta)(1 \leq \alpha<\beta \leq n)$ such that det $\sigma\left(\widetilde{P}_{l}\right)(t ; \tau) \neq 0$. This is a contradiction. Therefore this proves that 0 is not an eigenvalue of $\sigma\left(\widetilde{P}_{l}\right)(t ; \tau)$ under the condition $\sum_{k=1}^{n} t_{l k} \tau_{k 1} \neq 0$. This completes the proof of Lemma 2.6.

Q. E. D.

We can further verify the following.

Lemma 2.7. If a point $(t ; \tau)$ in $T^{*} X$ satisfies the condition (2.5.1), then $\pm \frac{2 \pi \sqrt{-1}}{\sqrt{2 \pi \sqrt{-1} \tau_{11}}} \sum_{k=1}^{n} t_{l k} \tau_{k l}$ are eigenvalues of $\sigma\left(\widetilde{P}_{l}\right)(t ; \tau)$.

Proof. Put

$$
\psi_{ \pm}(t ; \tau)=\left(\begin{array}{c}
\sqrt{2 \pi \sqrt{-1} \tau_{11}} \\
\pm 2 \pi \sqrt{-1} \tau_{11} \\
\vdots \\
\pm 2 \pi \sqrt{-1} \tau_{1 h} \\
\vdots \\
\pm 2 \pi \sqrt{-1} \tau_{1 n}
\end{array}\right)
$$


The column vectors $\phi_{ \pm}(t ; \tau)$ are eigenvectors of $\sigma\left(\widetilde{P}_{l}\right)(t ; \tau)$. In fact, we have

$$
\begin{aligned}
\sigma\left(P_{l}\right)(t ; \tau) \phi_{ \pm}(t ; \tau) & =2 \pi \sqrt{-1}\left(\begin{array}{c} 
\pm \sum_{k=1}^{n} t_{l k} \tau_{k 1} \\
\sqrt{2 \pi \sqrt{-1} \tau_{11}} \sum_{k=1}^{n} t_{l k} \tau_{k 1} \\
\vdots \\
\sqrt{2 \pi \sqrt{-1} \tau_{11}} \sum_{k=1}^{n} t_{l k} \tau_{k n}
\end{array}\right) \\
& =\left( \pm \frac{2 \pi \sqrt{-1}}{\sqrt{2 \pi \sqrt{-1} \tau_{11}}} \sum_{k=1}^{n} t_{l k} \tau_{k 1}\right) \psi_{ \pm}(t ; \tau) .
\end{aligned}
$$

Let us compute $\sigma\left(\left(\sqrt{2 \pi \sqrt{-1} \partial_{11}}\right)^{-1} R_{[\alpha, \beta]}\right)(t ; \tau) \phi_{ \pm}(t ; \tau)$. Since $(t ; \tau)$ satisfies the condition $(2.5 .1)$, we have

$$
\begin{aligned}
& \sigma\left(\left(\sqrt{2 \pi \sqrt{-1} \partial_{11}}\right)^{-1} R_{[\alpha, \beta]}\right)(t ; \tau) \phi_{ \pm}(t ; \tau) \\
& =\frac{2 \pi \sqrt{-1}}{\sqrt{2 \pi \sqrt{-1} \tau_{11}}}\left(\begin{array}{ccccccc}
0 & \cdots & 0 & \cdots & 0 & \cdots & 0 \\
0 & \cdots & -\tau_{\beta 1} & \cdots & \tau_{\alpha 1} & \cdots & 0 \\
\vdots & & \vdots & & \vdots & & \vdots \\
0 & \cdots & -\tau_{\beta h} & \cdots & \tau_{\alpha h} & \cdots & 0 \\
\vdots & & \vdots & & \vdots & & \vdots \\
0 & \cdots & -\tau_{\beta n} & \cdots & \tau_{\alpha n} & \cdots & 0
\end{array}\right)\left(\begin{array}{c}
\sqrt{2 \pi \sqrt{-1} \tau_{11}} \\
\pm 2 \pi \sqrt{-1} \tau_{11} \\
\vdots \\
\pm 2 \pi \sqrt{-1} \tau_{1 h} \\
\vdots \\
\pm 2 \pi \sqrt{-1} \tau_{1 n}
\end{array}\right) \\
& =0 .
\end{aligned}
$$

Hence we have

$$
\begin{aligned}
& \sigma\left(\widetilde{P}_{l}\right)(t ; \tau) \phi_{ \pm}(t ; \tau) \\
& \quad=\sigma\left(P_{l}\right)(t ; \tau) \phi_{ \pm}(t ; \tau)-\sum_{1 \leq \alpha<\beta \leq n} X(\alpha, \beta) \sigma\left(\left(\sqrt{2 \pi \sqrt{-1} \partial_{11}}\right)^{-1} R_{[\alpha, \beta]}\right) \phi_{ \pm}(\mathrm{t} ; \tau) \\
& \quad= \pm \frac{2 \pi \sqrt{-1}}{\sqrt{2 \pi \sqrt{-1} \tau_{11}}} \sum_{k=1}^{n} t_{l k} \tau_{k 1} \phi \pm(t ; \tau)
\end{aligned}
$$

Therefore $\pm \frac{2 \pi \sqrt{-1}}{\sqrt{2 \pi \sqrt{-1} \tau_{11}}} \sum_{k=1}^{n} t_{l k} \tau_{k 1}$ are eigenvalues of $\sigma\left(\widetilde{P}_{l}\right)(t ; \tau)$ and their eigenvectors are $\phi_{ \pm}$. This completes the proof of Lemma 2.7 .

Q. E. D.

Let us resume the proof of Proposition 2.5. Now suppose that a point $(t ; \tau)$ in $\mathrm{Ch}(K)$ satisfies the condition $\Re \sum_{k=1}^{n} t_{l k} \tau_{k 1} \neq 0$. Then by Lemma 2.6 and Lemma 2.7 the eigenvalues of $\sigma\left(\widetilde{P_{l}}\right)(t ; \tau)$ which do not depend on $X(\alpha, \beta)(1 \leq \alpha<\beta$ 
$\leq n)$ are $\pm \frac{2 \pi \sqrt{-1}}{\sqrt{2 \pi \sqrt{-1} \tau_{11}}} \sum_{k=1}^{n} t_{l k} \tau_{k 1}$, neither of which is purely imaginary. Furthermore, since the other eigenvalues of $\sigma\left(\widetilde{P}_{l}\right)(t ; \tau)$ depend holomorphically on generic $X(\alpha, \beta) \quad(1 \leq \alpha<\beta \leq n)$, we may assume that they are also in $\mathbf{C} \backslash \sqrt{-1} \mathbf{R}$ by choosing appropriate $X(\alpha, \beta) \quad(1 \leq \alpha<\beta \leq n)$. Hence by Lemma $2.4 \exp \widetilde{P}_{l}-I$ is invertible at $(t ; \tau)$. This proves

$$
\operatorname{Ch}(K) \subset\left\{(t ; \tau) \in T^{*} X \mid \Re \sum_{k=1}^{n} t_{l k} \tau_{k 1}=0 \quad \text { for } \quad 1 \leq l \leq n\right\}
$$

Expressing this relation in terms of the original numbering, we have

$$
\operatorname{Ch}(K) \subset\left\{(t ; \tau) \in T^{*} X \mid \Re \sum_{k=1}^{n} t_{l k} \tau_{k \imath}=0 \quad \text { for } \quad 1 \leq l \leq n\right\}
$$

For an arbitrary index $j$ in the same way we obtain

$$
\operatorname{Ch}(K) \subset\left\{(t ; \tau) \in T^{*} X \mid \Re \sum_{k=1}^{n} t_{l k} \tau_{k j}=0 \quad \text { for } \quad 1 \leq l \leq n\right\}
$$

if $\tau_{j j} \neq 0$. If $\tau_{j j}=0$, then (2.5.1) entails that $\tau_{k j}=0$ for $1 \leq_{k} \leq n$. Thus $(t ; \tau)$ satisfies

$$
\Re \sum_{k=1}^{n} t_{l k} \tau_{k \jmath}=0 \quad \text { for } \quad 1 \leq l \leq n
$$

Therefore this shows

$$
\operatorname{Ch}(K) \subset\left\{(t ; \tau) \in T^{*} X \mid \Re t \tau=0\right\}
$$

This completes the proof of Proposition 2.5.

Q. E. D.

\section{§3. Imaginary Transformation}

Throughout this section $X_{+}$denotes the domain

$$
X_{+}=\{t \in X \mid \Im t \text { is positive definite }\}
$$

As Proposition $2.5(2.5 .4)$ implies that a point $(t ; \tau)$ in $\mathrm{Ch}(K)$ satisfies det $\Im t$ $=0$ if $\tau \neq 0$, we find 


$$
\left.\operatorname{Ch}(K) \cap T^{*} X\right|_{X_{+}} \subset T_{X}^{*} X
$$

where $T_{X}^{*} X$ denotes the zero-section of $T^{*} X$. It then follows from Theorem 4.1.1 of [5] that any germ of holomorphic solution of the system $K$ at $t$ in $X_{+}$is $\mathrm{u}_{\text {- }}$ niquely extended over $X_{+}$. Furthermore Theorem 2.2 combined with Remark 2.16 of [8] shows that any holomorphic solution of $K$ on $X_{+}$is a constant multiple of $(\vartheta(t), 0, \cdots, 0)^{\prime}$. On the other hand the domain $X_{+}$admits an automorphism $\phi$ given by $t \mapsto-t^{-1}$. In fact, one can readily verify that det $t \neq 0$ and $-t^{-1}$ is in $X_{+}$if $t$ is in $X_{+}$. We shall now study how this system $K$ will be changed by the transformation $\phi$ and study how the one-dimensionality result for the holomorphic solution of $K$ on $X_{+}$can be used to deduce the automorphic property of $\vartheta(t)$ under the map $\phi$ : To do this, we set $s=-t^{-1}$ and for a differential operator $L$ on $X_{+}$we define a differential operator $\widetilde{L}$ by the following:

$$
\begin{aligned}
\widetilde{L f}(s) & \stackrel{\text { def }}{=}\left(\phi^{*} L\right) f(s) \\
& =(L f)(s)
\end{aligned}
$$

with $f(s)$ being an arbitrary function. Noting that $X_{+}$is simply-connected, we have a single-valued function (det $t)^{\frac{1}{2}}$ by determining its value at

$$
t_{0}=\left(\begin{array}{ccc}
\sqrt{-1} & & 0 \\
& \ddots & \\
0 & & \sqrt{-1}
\end{array}\right)
$$

to be $\exp \frac{n \pi \sqrt{-1}}{4}$. Now let us introduce the following $(n+1) \times(n+1)$ matrix $T$ :

$$
(\operatorname{det} t)^{\frac{1}{2}}\left(\begin{array}{cccc}
1 & 0 & \cdots & 0 \\
0 & t_{11} & \cdots & t_{1 n} \\
\vdots & \vdots & & \vdots \\
0 & t_{n 1} & \cdots & t_{n n}
\end{array}\right) .
$$

Using this matrix $T$, we find the following relations among $\widetilde{P}_{l}$ 's, $\widetilde{Q}_{m}$ 's and $P_{l}$ 's, $Q_{m}$ 's, generalizing the result of [4] in the higher dimensional case. The following theorem further shows that $R_{[l, m]} u=0$ and $S_{[k, l, m]} u=0$ are kept essentially intact.

Theorem 3.1 The matrix $T$ is invertible on the domain $X_{+}$and we find the following relations on $X_{+}$: 


$$
T^{-1} \widetilde{P}_{l} T=-Q_{l},
$$

$$
\begin{aligned}
T^{-1} \widetilde{Q}_{l} T & =P_{l}, \\
T^{-1} \widetilde{R}_{[l, m]} T & =\sum_{i, j=1}^{n} t_{l i} t_{m j} R_{[\imath, j]}, \\
T^{-1} \widetilde{S}_{[\alpha, l, m]} T & =\sum_{i, j, k=1}^{n} t_{\alpha k} t_{l i} t_{m j} S_{[k, i, j]},
\end{aligned}
$$

for $1 \leq \alpha, l, m \leq n$.

Proof. Denote $-t^{-1}$ by $s=\left(s_{i j}\right)_{1 \leq i, j \leq n}$. We then find

$$
T^{-1}=\frac{1}{(\operatorname{det} t)^{\frac{1}{2}}}\left(\begin{array}{cccc}
1 & 0 & \cdots & 0 \\
0 & -s_{11} & \cdots & -s_{1 n} \\
\vdots & \vdots & & \vdots \\
0 & -s_{n 1} & \cdots & -s_{n n}
\end{array}\right) .
$$

By using the relations

$$
\frac{\partial s}{\partial t_{i j}}=t^{-1}\left(1+\delta_{i j}\right)^{-1}\left(E_{\imath j}+E_{j_{\imath}}\right) t^{-1},
$$

we also find

$$
\partial_{i j} s_{k l}=s_{k j} s_{\imath l}+s_{k l} s_{j l}
$$

for $1 \leq i, j, l, k \leq n$. Then we find

$$
\begin{aligned}
\frac{\partial}{\partial s_{i j}}(\operatorname{det} t)^{\frac{1}{2}} & =\frac{1}{2}(\operatorname{det} t)^{\frac{1}{2}}(\operatorname{det} t)^{-1} \frac{\partial}{\partial s_{i j}} \operatorname{det} t \\
& =\frac{(-1)^{n}}{2}(\operatorname{det} t)^{\frac{1}{2}}(\operatorname{det} t)^{-1} \frac{\partial}{\partial s_{i j}}(\operatorname{det} s)^{-1} \\
& =\frac{-(-1)^{n}}{2}(\operatorname{det} t)^{\frac{1}{2}}(\operatorname{det} t)^{-1}(\operatorname{det} s)^{-2} \frac{\partial}{\partial s_{i j}} \operatorname{det} s
\end{aligned}
$$

On the other hand we have the following relation:

$$
\begin{aligned}
(\operatorname{det} s)^{-1} \frac{\partial}{\partial s_{i j}} \operatorname{det} s & =(\operatorname{det} s)^{-1}\left(1+\delta_{i j}\right)^{-1}\left(\Delta_{\imath j}+\Delta_{j i}\right) \\
& =-\left(1+\delta_{i j}\right)^{-1}\left(\mathrm{t}_{i j}+\mathrm{t}_{j_{\imath}}\right)
\end{aligned}
$$


for $1 \leq i, j \leq n$, where $\Delta_{i j}$ denotes the matrix obtained from $s$ by deleting its $i$-th row and $j$-th column, i.e.,

$$
(-1)^{i+j} \operatorname{det}\left(\begin{array}{cccccc}
s_{11} & \cdots & s_{1 j-1} & s_{1 j+1} & \cdots & s_{1 n} \\
\vdots & & \vdots & \vdots & & \vdots \\
s_{i-1,1} & \cdots & s_{i-1, j-1} & s_{i-1, j+1} & \cdots & s_{i-1, n} \\
s_{i+1,1} & \cdots & s_{i+1, j-1} & s_{i+1, j+1} & \cdots & s_{i+1, n} \\
\vdots & & \vdots & \vdots & & \vdots \\
s_{n 1} & \cdots & s_{n, j-1} & s_{n, j+1} & \cdots & s_{n n}
\end{array}\right) .
$$

Making use of (44), we deduce from (43)

$$
\widetilde{\partial}_{i j}(\operatorname{det} t)^{\frac{1}{2}}=(\operatorname{det} t)^{\frac{1}{2}} t_{i j}
$$

We first note that each entry of the matrix $\widetilde{P}_{l} T$ is as follows:

$$
\begin{array}{rlrl}
\left(\widetilde{P}_{l} T\right)_{i+1, j+1} & =(\operatorname{det} t)^{\frac{1}{2}} \sum_{k=1}^{n} s_{l k} t_{k j} & \text { if } \quad & i=0,1 \leq j \leq n, \\
& =2 \pi \sqrt{-1}(\operatorname{det} t)^{\frac{1}{2}}\left(\delta_{l \imath}+\sum_{k=1}^{n} s_{l k} \widetilde{\partial}_{k \imath}\right)+2 \pi \sqrt{-1} \sum_{k=1}^{n} s_{l k} \widetilde{\partial}_{k l}(\operatorname{det} t)^{\frac{1}{2}} \\
& =0 & & \text { if } 1 \leq i \leq n, \mathrm{j}=0, \\
& & \text { otherwise. }
\end{array}
$$

Using (45), we deduce from (47)

$$
\begin{aligned}
\left(\widetilde{P}_{l} T\right)_{i+1,1} & =2 \pi \sqrt{-1}(\operatorname{det} t)^{\frac{1}{2}}\left(\delta_{l \imath}+\sum_{k=1}^{n} s_{l k} \widetilde{\partial}_{k i}+\sum_{k=1}^{n} s_{l k} \mathrm{t}_{k \imath}\right) \\
& =2 \pi \sqrt{-1}(\operatorname{det} t)^{\frac{1}{2}} \sum_{k=1}^{n} s_{l k} \widetilde{\partial}_{k i}
\end{aligned}
$$

for $i \geq 1$. On the other hand it immediately follows that

$$
\left(\widetilde{P}_{l} T\right)_{1, j+1}=-(\operatorname{det} t)^{\frac{1}{2}} \delta_{l j}
$$

for $j \geq 1$. Therefore we have 


$$
\widetilde{P}_{l} T=(\operatorname{det} t)^{\frac{1}{2}}\left(\begin{array}{cccccc}
0 & -\delta_{l 1} & \cdots & -\delta_{l j} & \cdots & -\delta_{l n} \\
2 \pi \sqrt{-1} \sum_{k=1}^{n} s_{l k} \widetilde{\partial}_{k 1} & 0 & \cdots & 0 & \cdots & 0 \\
\vdots & \vdots & & \vdots & & \vdots \\
2 \pi \sqrt{-1} \sum_{k=1}^{n} s_{l k} \widetilde{\partial}_{k l} & 0 & \cdots & 0 & \cdots & 0 \\
\vdots & \vdots & & \vdots & & \vdots \\
2 \pi \sqrt{-1} \sum_{k=1}^{n} s_{l k} \widetilde{\partial}_{k n} & 0 & \cdots & 0 & \cdots & 0
\end{array}\right) .
$$

Hence we find

$$
T^{-1} \widetilde{P}_{l} T=\left(\begin{array}{cccccc}
0 & -\delta_{l 1} & \cdots & -\delta_{l j} & \cdots & -\delta_{l n} \\
-2 \pi \sqrt{-1} \sum_{k, m=1}^{n} s_{1 m} s_{l k} \widetilde{\partial}_{k m} & 0 & \cdots & 0 & \cdots & 0 \\
\vdots & \vdots & & \vdots & & \vdots \\
-2 \pi \sqrt{-1} \sum_{k, m=1}^{n} s_{l m} s_{l k} \widetilde{\partial}_{k m} & 0 & \cdots & 0 & \cdots & 0 \\
\vdots & \vdots & & \vdots & & \vdots \\
-2 \pi \sqrt{-1} \sum_{k, m=1}^{n} s_{n m} s_{l k} \widetilde{\partial}_{k m} & 0 & \cdots & 0 & \cdots & 0
\end{array}\right) .
$$

Using (42), we deduce from the $(i+1,1)$-component of the matrix $T^{-1} \widetilde{P}_{l} T$

$$
\begin{aligned}
\left(T^{-1} \widetilde{P}_{l} T\right)_{\imath+1,1} & =-2 \pi \sqrt{-1} \sum_{k \geq m}\left(s_{i m} s_{l k}+s_{\imath k} s_{l m}\right) \frac{\partial}{\partial s_{k m}} \\
& =-2 \pi \sqrt{-1}\left(1+\delta_{i l}\right) \sum_{k \geq m} \frac{\partial s_{k m}}{\partial t_{\imath l}} \frac{\partial}{\partial s_{k m}} \\
& =-2 \pi \sqrt{-1} \partial_{\imath l}
\end{aligned}
$$

for $i \geq 1$. Hence we have $T^{-1} \widetilde{P}_{l} T=-Q_{l}$ for $1 \leq l \leq n$. This proves (38).

To verify (39) we notice that each entry of the matrix $P_{l} T^{-1}$ is given as follows:

$$
\begin{aligned}
& \left(P_{l} T^{-1}\right)_{i+1, j+1}=-(\operatorname{det} t)^{-\frac{1}{2}} \sum_{k=1}^{n} t_{l k} s_{k j} \quad \text { if } \quad i=0,1 \leq_{j} \leq_{n} \text {, } \\
& =2 \pi \sqrt{-1}(\operatorname{det} t)^{-\frac{1}{2}}\left(\delta_{l \imath}+\sum_{k=1}^{n} t_{l k} \partial_{k \imath}\right)+2 \pi \sqrt{-1} \sum_{k=1}^{n} t_{l k} \partial_{k l}(\operatorname{det} t)^{-\frac{1}{2}} \\
& =0 \quad \text { if } 1 \leq i \leq n, \mathrm{j}=0 \text {, } \\
& \text { otherwise. }
\end{aligned}
$$

In the same way with $T^{-1} \widetilde{P}_{l} T$, we have 


$$
\begin{aligned}
T P_{l} T^{-1} & =\left(\begin{array}{ccccc}
0 & & \delta_{l 1} & \cdots & \delta_{l n} \\
2 \pi \sqrt{-1} \sum_{k, m=1}^{n} & t_{1 m} t_{l k} \partial_{k m} & 0 & \cdots & 0 \\
\vdots & & \vdots & & \vdots \\
2 \pi \sqrt{-1} \sum_{k, m=1}^{n} & t_{i m} t_{l k} \partial_{k m} & 0 & \cdots & 0 \\
\vdots & & \vdots & & \vdots \\
2 \pi \sqrt{-1} \sum_{k, m=1}^{n} & t_{n m} t_{l k} \partial_{k m} & 0 & \cdots & 0
\end{array}\right) \\
& =\left(\begin{array}{cccc}
0 & \delta_{l 1} & \cdots & \delta_{l n} \\
2 \pi \sqrt{-1} \tilde{\partial}_{l 1} & 0 & \cdots & 0 \\
\vdots & \vdots & & \vdots \\
2 \pi \sqrt{-1} \tilde{\partial}_{l i} & 0 & \cdots & 0 \\
\vdots & \vdots & & \vdots \\
2 \pi \sqrt{-1} \tilde{\partial}_{l n} & 0 & \cdots & 0
\end{array}\right) \\
& =\widetilde{Q}_{l .} \\
&
\end{aligned}
$$

Then it entails $T^{-1} \widetilde{Q}_{l} T=P_{l}$.

To prove (40) we use Theorem 2.2 together with the computation given above. Then we have

$$
\begin{aligned}
T^{-1} \widetilde{R}_{[l, m]} T & =T^{-1}\left[\widetilde{Q}_{l}, \widetilde{Q}_{m}\right] T \\
& =\left[P_{l}, P_{m}\right] \\
& =\sum_{i, j=1}^{n} t_{l i} t_{m j} R_{[i, j]} .
\end{aligned}
$$

This proves (40). The verification of (41) can be done in a similar manner; we have

$$
\begin{aligned}
T^{-1}{\widetilde{S_{[\alpha, l, m]}}} & =T^{-1}\left[\widetilde{Q}_{\alpha}, \widetilde{R}_{[l, m]}\right] T \\
& =\left[P_{\alpha}, \sum_{i, j=1}^{n} t_{l i} t_{m j} R_{[i, j]}\right] \\
& =\sum_{i, j=1}^{n}\left[P_{\alpha}, t_{l i} t_{m j} I\right] R_{[\imath, j]}+\sum_{i, j=1}^{n} t_{l i} t_{m j}\left[P_{\alpha}, R_{[i, j]}\right]
\end{aligned}
$$

On the other hand we have

$$
\left[P_{\alpha}, t_{l i} t_{m j} I\right] R_{[i, j]}=0
$$


in fact all components in $\left[P_{\alpha}, t_{l i} t_{m j} I\right]$ vanish except for the first column and such a matrix annihilates $R_{[i, j]}$. By Theorem 2.2 we deduce from (49)

$$
T^{-1} \widetilde{S}_{[\alpha, l, m]} T=\sum_{i, j=1}^{n} t_{l i} t_{m j} \sum_{k=1}^{n} t_{\alpha k} S_{[k, i, j]}
$$

This completes the proof.

Q.E. D.

As $T^{-1} \exp \widetilde{P}_{l} \cdot T=\exp \left(T^{-1} \widetilde{P}_{l} T\right)$ etc. hold, Theorem 3.1 implies that both $T^{-1}\left(\vartheta\left(-t^{-1}\right), 0, \cdots, 0\right)^{\prime}$ and $(\vartheta(t), 0, \cdots, 0)^{\prime}$ satisfy the following equations:

$$
\begin{gathered}
T^{-1}\left(\exp \widetilde{P}_{l}-I\right) T u=0, \\
T^{-1}\left(\exp \widetilde{Q}_{m}-I\right) T u=0, \\
T^{-1} \widetilde{R}_{[l, m]} T u=0, \\
T^{-1} \widetilde{S}_{[k, l, m]} T u=0,
\end{gathered}
$$

for $1 \leq k, l, m \leq n$. Since the space of holomorphic solutions of (50), (51), (52) and (53) on $X_{+}$is 1 -dimensional, there exists a constant $C$ such that

$$
\left(\begin{array}{c}
\vartheta(t) \\
0 \\
\vdots \\
0
\end{array}\right)=C T^{-1}\left(\begin{array}{c}
\vartheta\left(-t^{-1}\right) \\
0 \\
\vdots \\
0
\end{array}\right)
$$

Comparing the both sides of (54) at $t_{0}$, we have

$$
\begin{aligned}
C & =\left(\operatorname{det} t_{0}\right)^{\frac{1}{2}} \\
& =\exp \frac{n \pi \sqrt{-1}}{4} .
\end{aligned}
$$

Hence we have $\vartheta(t)=\exp \frac{n \pi \sqrt{-1}}{4} \cdot(\operatorname{det} t)^{-\frac{1}{2}} \vartheta\left(-\mathrm{t}^{-1}\right)$.

\section{References}

[1] Aoki, T., Invertibility for microdifferential operators of infinite order, Publ. RIMS. Kyoto Univ., 18 (1982), 421-449.

[2] Aoki, T., Kashiwara, M. and Kawai, T., On a class of linear differntial operators of infinite order with finite index, Adv. in Math., 62 (1986), 155-168. 
[3] Berenstein, C. A., Kawai, T., Struppa, C. and Takei, Y., Exponential representation of a holomorophic solution of a system of differential equations associated with the theta-zerovalue, preprint.

[4] Kashiwara. M., Kawai, T. and Takei, Y., The structure of cohomology groups associated with the theta-zerovalues, Proceedings of Geometrical and Algebraical Aspect in Several Complex Variables (eds. Berenstein-Struppa), Cetraro, (1989), 169-189.

[5] Kashiwara, M. and Schapira, P., Micro-hyperbolic systems, Acta Math., 142 (1979), 1-55.

[6] Kawai, T., Some remarks on the microlocal analysis of $\vartheta$-function, RIMS Kôkyûroku, Kyoto Univ., No. 410, (1980), 76-87.

[7] Sato, M., Pseudo-differential equations and theta functions, Astérisque 2 et 3 (1973), 286291.

[8] Sato, M., Kashiwara, M. and Kawai, T., Linear differential equations of infinite order and theta functions, Adv. in Math., 47 (1983), 300-325.

[9] Microlocal analysis of theta functions, Advanced Studies in Pure Math., 4 (1984), $267-289$.

[10] - Microfunctions and pseudo-differntial equations, Lecture Note in Math., Springer, Berlin-Heidelberg-New York, 287 (1973), 289-529. 